\title{
STUDIES ON RENAL TUBULAR TRANSPORT
}

\section{ACCUMULATION OF P-AMINOHIPPURATE BY KIDNEY SLICES*}

\author{
TETSUO KOISHI \\ Department of Pharmacology, Kyoto Prefectural Medical College, Kyoto
}

Received for publication June 6, 1958

\section{INTRODUCTION}

In the past few years, various functions of kidney have been studied, and their mechanisms have been elucidated step by step, with improved techniques, such as the clearance method and the puncture method applied to glomerulus and renal tubulus. Among the functions of renal tubulus, the transport mechanism, such as reabsorption of glucose and water, and regulation of electrolytes, especially, exchange of $\mathrm{Na}^{+}, \mathrm{H}^{+}$, and $\mathrm{HCO}_{3}^{-}$, and the synthesis of ammonia have been understood almost satisfactorily, while the study on the transport of foreign substance has been undertaken by few workers.

The mechanism of transport of foreign substance might be different among the variety of transported substances and animal species. One of these foreign substances, para-aminohippurate (briefly, PAH) is excreted through glomerulus and tubulus, as phenol red or diodrast is. And PAH is excreted completely when blood passes through kidney, without reabsorption. Smith and his collaborators (1) have established a method of estimation of renal plasma flow (RPF) by the clearance of PAH, and applied it to human body. This is an example to show that PAH and phenol red are suitable substances to estimate RPF and to observe a feature of excretion of foreign substance.

Recently, Forster (2) has observed microscopically that kidney slices accumulate phenol red, out of medium containing it. Beyer et al. $(3,4)$ have studied the effect of inhibitors using the technique of Forster and concluded that phenol red transport is performed by excretion through tubulus cells and it was closely related with energy-rich phosphate bonds. Cross and Taggart (5), having modified the method of Forster, have observed that PAH is accumulated by kidney slices against its concentration gradient, when the slices are incubated in the medium containing PAH of given concentrations, and studied the effect of a number of reagents on the PAH accumulation. They have considered that acetate might be a rate-limiting cellular component of PAH transport mechanism, based upon the observation of the stimulatory effect of acetate on the accumulation. It has also been established that these results of PAH excretion in vivo are well consistent with the results of PAH accumulation in vitro, and the PAH accumulation is specific to kidney slice (5,

* Director : Prof. Dr. S. Kozawa 
6). The effects of 2,4-dinitrophenol (7), fluoroacetate (8), and various electrolytes (9) in media on the PAH accumulation have been examined and the correlation between incubation time and $\mathrm{pH}$ of medium has been detected (10). In these experiments, emphasis has been placed on the stimulatory effect of acetate on the PAH accumulation $(6,11,12)$, however, the mechanism of PAH transport and the effect of acetate on it has been remained stil to be solved.

The present investigation was undertaken to see the effects of various reagents on the transport of foreign substance, following the method of Cross \& Taggart (5), and the results obtained and the explanations undertaken differed from theirs in some respects. In addition, on the basis of these results, the effects of a number of members in citric acid cycle and related substances have been observed, and their mechanism has been treated from an enzymological view point.

\section{EXPERIMENT}

Kidney slices were used as material, and incubated in the Warburg apparatus.

1) Reagents: The medium in the main compartment was prepared according to the directions of Cross \& Taggart (5), that is :

\begin{tabular}{|c|c|c|}
\hline $0.3 \mathrm{M}$ & Sodium chloride & 0.8 \\
\hline $0.1 \mathrm{M}$ & Phosphate buffer $(\mathrm{pH}=7.4)$ & 0.2 \\
\hline $3 \mathrm{~N}$ & Potassium chloride & 0.3 \\
\hline $0.02 \mathrm{M}$ & Calcium chloride & 0.1 \\
\hline$M$ & Para-aminohippurate (PAH) & 0.2 \\
\hline
\end{tabular}

The concentration of PAH was increased up to $0.01 \mathrm{M}$, when the alteration was necessary.

The side arm was equipped with 0.3 c.c. of the solution which contains the substrate in citric acid cycle or the related substance, mainly as the sodium salt.

2) Material: Rat was used. Decapitating it, the kidneys were removed and chilled promptly in ice-cold $0.15 \mathrm{~N}-\mathrm{NaCl}$. In the solution, slices of $0.3-0.5 \mathrm{~mm}$ thickness were cut, after removing away the kidney fatty capsule, and their cortex portions were separated out and used.

3) Procedures: About $100 \mathrm{mg}$ of slices were weighed on a torsion balance, and were rapidly added to the main compartment which contained 2.7 c.c. of above cited medium and had been cooled in cracked ice. The side arm was equipped with substrate, and the center well contained $15 \% \mathrm{KOH}$, as absorbent of $\mathrm{CO}_{2}$. The vessel was rapidly set on the Warburg manometer and the gas space was filled with oxygen. Then, it was shaken in a $37.5^{\circ} \mathrm{C}$ thermostat at a rate of 120 cycles/minute. After 10 minutes of shaking, contents of the main compartment and the side arm were mixed altogether. After 15 minutes of shaking, the oxygen uptake was recorded during the following 60 minutes of incubation.

The incubation was continued for about 10 minutes moreover, and the vessel was removed without ceasing the shaking, and chilled immediately in an ice-box. The content 
of the vessel was poured into an ice-cold dish, the slices were lifted and blotted carefully on filter paper, and weighed on a torsion balance, to which $5 \%$ trichloroacetic acid was added to a final volume of 10.0 c.c. (or 8.0 c.c. in some cases). The slices, thereafter, were broken up with stirring rod of glass. To a 1.0 c.c. aliquot of the slightly turbid suspending medium, 5\% trichloroacetic acid was also added, up to a final volume of 10.0 c.c. The both solutions prepared were filtered after 20 minutes, and 5.0 c.c. aliquots of the filtrate were used for the estimation of PAH.

4) Estimation of PAH: The procedures of Bratton \& Marshall (13) were followed. The protein-free filtrate was prepared with trichloroacetic acid as mentioned before, to 5.0 c.c. of which were added 0.5 c.c. of $0.1 \mathrm{~g} / \mathrm{d} L$ sodium nitrite, after 4 minutes 0.5 c.c. of $0.5 \mathrm{~g} / \mathrm{d} L$ ammonium sulfamate, and after 4 minutes 0.5 c.c. of $0.1 \mathrm{~g} / \mathrm{d} L \mathrm{~N}$-(1-naphthyl)-ethylenediamine dihydrochloride. Then, after 10 minutes equilibration, distilled water was added to the final volume of 10.0 c.c. and the PAH content of the solution was determined by a photoelectric colorimeter equipped with green filter.

The concentration of the accumulated PAH was expressed on the final wet weight basis, while the oxygen uptake was represented on the basis of the initial wet weight, because some of cell components were thought to disperse into the medium and consume oxygen, though they were excluded from the final wet weight of slices.

\section{RESULTS}

\section{Relation Between Total Amount of PAH and Its Concentration in Slices}

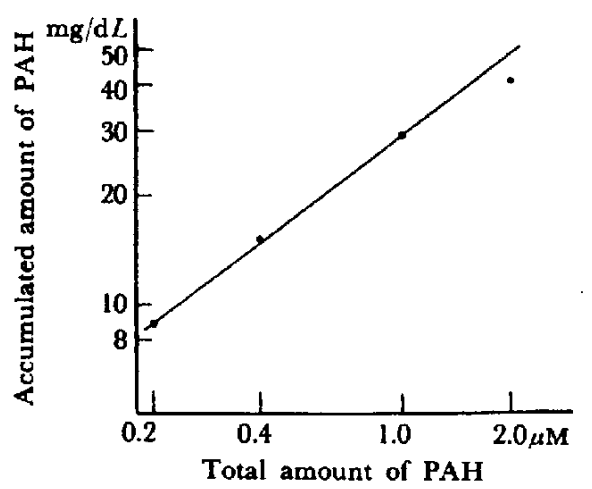

Fig. 1. Effect of total amount of PAH in the vessel on the accumulated amount in the slices. $\left(37.5^{\circ} \mathrm{C}, 120\right.$ cycles/minute)

For the preliminary observation, concentration of $\mathrm{PAH}$ accumulated in slices was determined, under various total amounts of PAH in the vessel, that is, 0.2, 0.4, 1.0, and $2.0 \mu \mathrm{M}$ of $\mathrm{PAH}$, at $37.5^{\circ} \mathrm{C}$ and 120 cycles/ minute. The result is shown in Fig. 1, where the mean values of every 8 cases are plotted according to Freundlich equation. The vertical axis represents logarithm of concentration of the accumulated PAH and horizontal axis that of total amount of PAH in the main compartment.

The plot gives a straight line, in accordance with the data obtained by Cross \& Taggart at $25^{\circ} \mathrm{C}$ and 100 cycles/minute.

\section{Effect of Pyruvate on the Accumulation of PAH}

Since PAH is accumulated by the kidney slices out of the medium against its concentration gradient, it is convenient to indicate the accumulative potent of slices by $\mathrm{S} / \mathrm{M}$ ratio, where $S$ and $M$ represent the concentration of $P A H$ in the slices and the medium 


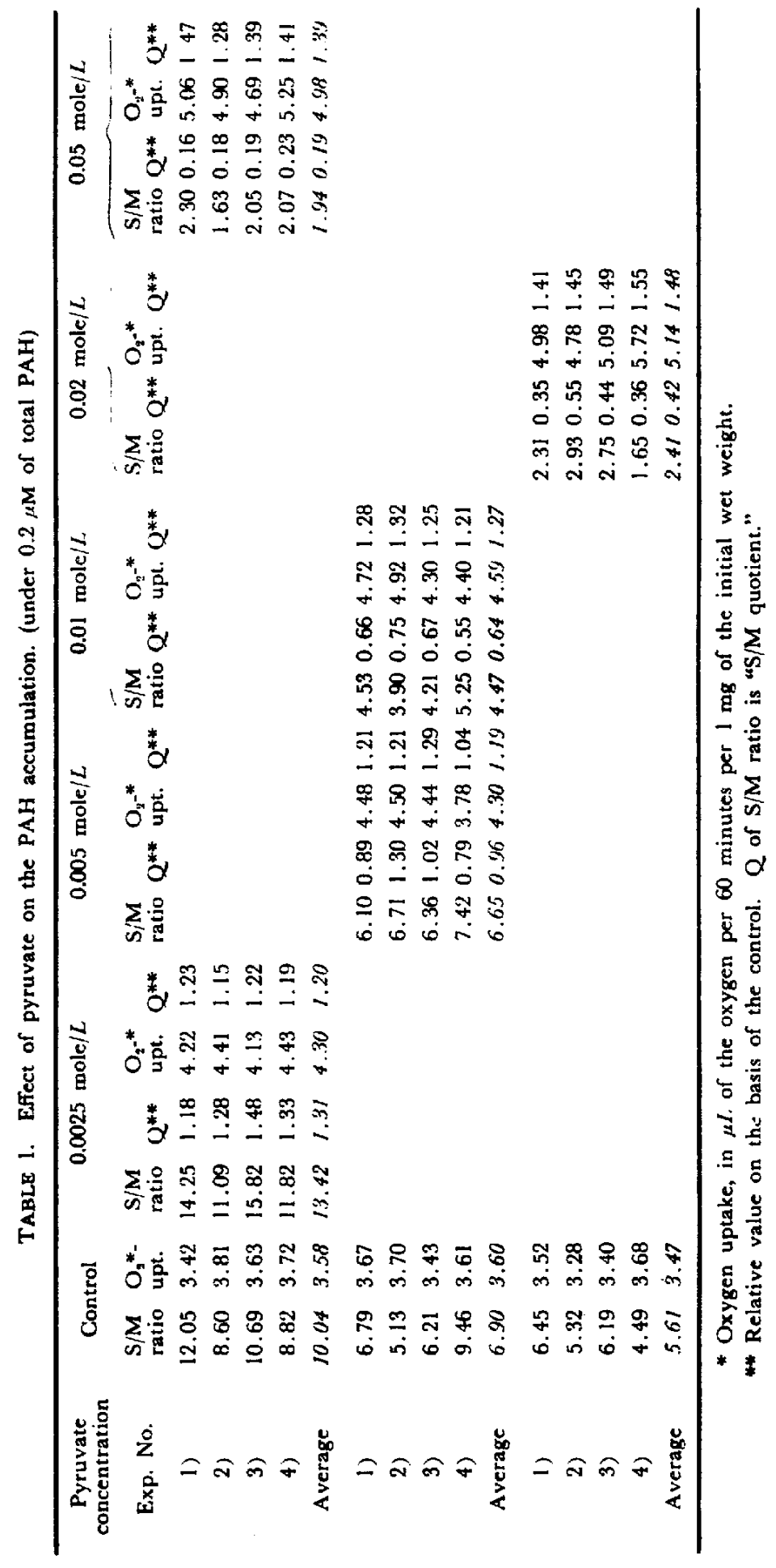


respectively. And to minimize the relatively large variation among individual materials, $\mathrm{S} / \mathrm{M}$ quotient, which was defined as the relative value of $\mathrm{S} / \mathrm{M}$ ratio on the basis of that of the control determined at the same time, might be suitable. The data shown in the following tables are recorded in terms of $S / M$ ratio and $S / M$ quotient, and those shown in the figures are in terms of $S / M$ quotient.

I) Effect of pyruiate concentration: The accumulation of PAH was observed under various concentrations of pyruvate and $0.2 \mu \mathrm{M}$ of total PAH. at $37.5 \mathrm{C}$ and 120 cycles/minute. The result is shown in Table 1 . The $S / M$ quotient decreases with increasing concentration of pyruvate, and it becomes smaller than unity at the concentration as low as 0.01 mole $/ L$

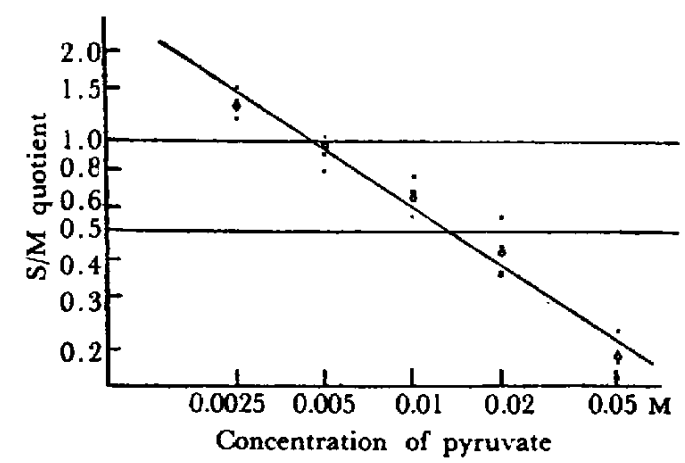

FIG. 2. Effect of pyruvate on the $\mathrm{S} / \mathrm{M}$ quotient. $\log y=-1.519-0.648 \log x$ (total pyruvate in the vessel being 30 $\mu \mathrm{M})$, taken by the previous workers usually, showing obvious inhibition. In Fig. 2, the $\mathrm{S} / \mathrm{M}$ quotient has been plotted against the concentration of pyruvate in logarithmic scales. The plot gives a straight line, and the best straight line was estimated by least square methods (14).

The residual variance has been tested by the usual manner of statistics and the null hypothesis of linear regression was not rejected, at $5 \%$ level of significance. These statistical procedures have been adopted to obtain all the experimental equations given in this paper, while the repetition of the statement will be omitted for the sake of brevity.

In addition, the initial $\mathrm{pH}$ was in the range of 7.2 to 7.4 , and it was not affected by pyruvate. The final $\mathrm{pH}$, that was obtained after the incubation for 90 minutes at $37.5^{\circ} \mathrm{C}$, did not differ markedly from the initial one. Both of these values fell into the range of 7.0 to 7.8 where none of effect on the PAH accumulation has been disclosed.

2) Effects of bath temperature and frequency of shaking: The accumulation of PAH was observed at a temperature of $25^{\circ} \mathrm{C}$ and a shaking rate of $100 \mathrm{cycles} / \mathrm{minute}$. The result obtained is given in Table 2. The $\mathrm{S} / \mathrm{M}$ quotient is 1.3 at $0.01 \mathrm{~mole} / L$ pyruvate $(30 \mu \mathrm{M}$ of total amount), which is in accordance with the data of Cross et al. (5). However, the

TABLE 2. Effect of temperature on $\mathrm{S} / \mathrm{M}$ quotient. (Average of 4 cases)

\begin{tabular}{ccc}
\hline $\begin{array}{c}\text { Pyruvate } \\
\text { concentration } \\
\text { (mole } L \text { ) }\end{array}$ & $25.0^{\circ} \mathrm{C}$ & $37.5^{\circ} \mathrm{C}$ \\
\hline 0.005 & 1.57 & 0.96 \\
0.01 & 1.30 & 0.64 \\
0.02 & 1.07 & 0.42 \\
0.05 & 0.73 & 0.19 \\
\hline
\end{tabular}
quotient decreases, though slowly, with increasing pyruvate, as shown in the case above mentioned. The data fit Freundlich equation as shown in Fig. 3, and it is seen that Freundlich equation is always obeyed under some width of range of temperatures and shaking rates.

3) Relation belween concentrations of pyruvate and PAH: It is natural to interpret above mentioned data as showing that the action of pyruvate or its metabolic product 
is inhibitory as well as stimulatory.

In order to observe these two contradicting activities in detail, experiment (1) was repeated under various concentrations of PAH in media, that is, these were taken to be 2, 5 , and 10 times as large as the concentration tested before $(0.4,1.0$, and $2.0 \mu \mathrm{M}$ of total amount respectively). The plot according to Freundlich equation is given in Fig. 4, together with the plot of experiment (1), where each point of the former represents average value of 4 cases. The slope decreases and the intercept increases, with increasing concentration of PAH. And these plots show that the inhibition by pyruvate is suppressed and the stimulation by it is improved, with increasing PAH concentration.

4) Fifty percent inhibition diagram: According to the above mentioned data, it is obvious

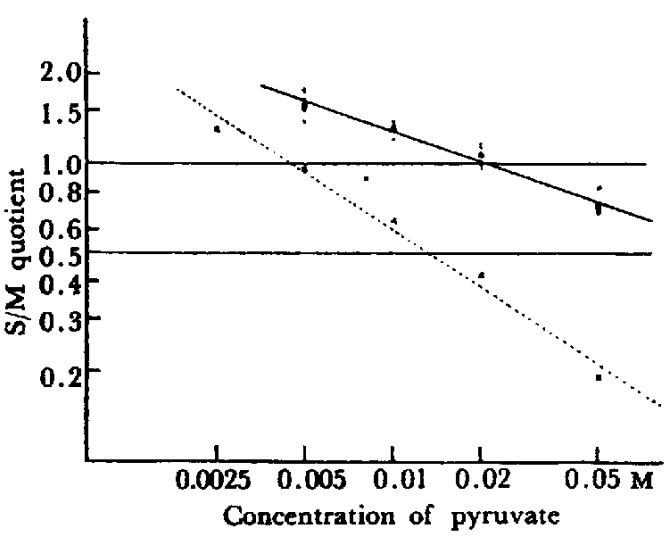

FIG. 3. Effect of temperature on the $S / M$ quotient.

$$
\begin{aligned}
& 25^{\circ} \mathrm{C}(\mathrm{O}: \text { Average }): \\
& \log \mathrm{y}=-0.559-0.333 \log \mathrm{x} \\
\ldots . .57 .5^{\circ} \mathrm{C}: & \\
& \log \mathrm{y}=-1.519-0.648 \log \mathrm{x}
\end{aligned}
$$

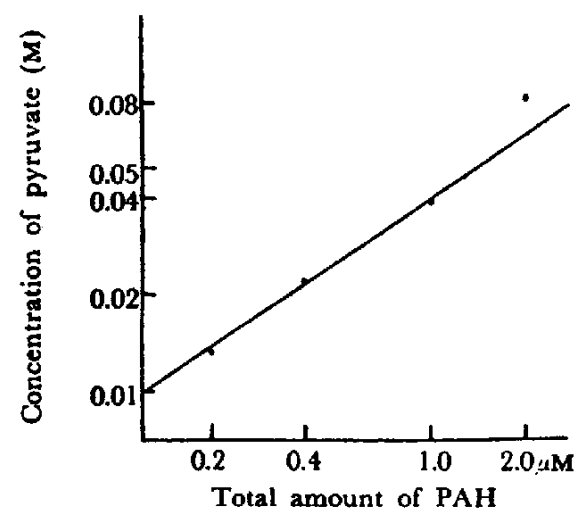

FIG. 5. Fifty percent inbibition diagram. Note: The lines were obtained by weighting each of the mean values. $\log y=2.560 \rightarrow 0.660 \log x$ (weighted line)

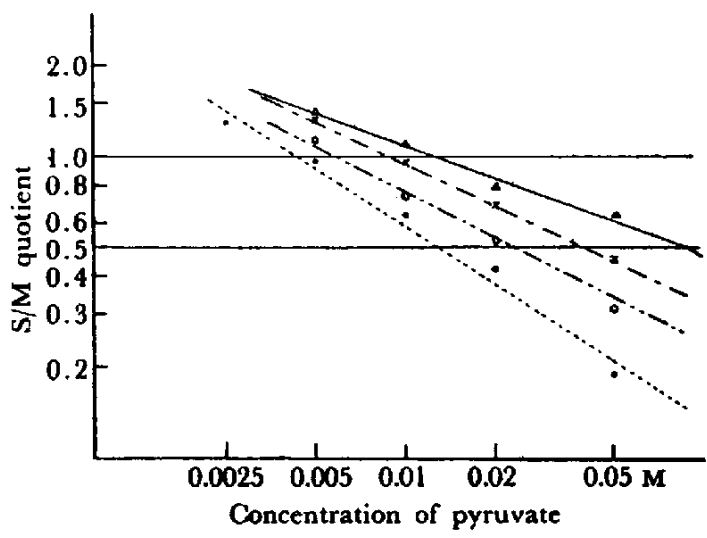

FIG. 4. Effect of pyruvate on the $S / M$ quotient under various total amount of PAH.

- ..... $0.2 \mu \mathrm{M}$ of total PAH : $\log y=-1.519-0.648 \log x$

c - $00.4 \mu \mathrm{M}$ of total PAH : $\log y=-1.104-0.492 \log x$

$x---x 1.0 \mu \mathrm{M}$ of total PAH : $\log y=-0.955-0.466 \log x$

$\triangle \longrightarrow \triangle 2.0 \mu \mathrm{M}$ of total PAH :

$\log y=-0.681-0.354 \log x$

TABLE 3. Fifty percent inhibition concentration of pyruvate.

\begin{tabular}{cc}
\hline $\begin{array}{c}\text { Total PAH } \\
(\mu \mathrm{M})\end{array}$ & $\begin{array}{c}\text { 50\%o inhibition } \\
\text { concentration (mole } / L)\end{array}$ \\
\hline 0.2 & 0.0132 \\
0.4 & 0.0234 \\
1.0 & 0.0394 \\
2.0 & 0.0845 \\
\hline
\end{tabular}


that the extent of inhibition depends on both PAH and pyruvate, and the inhibitory effect of pyruvate or its metabolic products is competitive with PAH for the accumulation by the slices. The feature of the competition is shown graphically in Fig. 5 (50\% inhibition diagram), where the pyruvate concentration which decreases $S / M$ ratio down to $50 \%$ of the control value, that is, S/M quotient to a half, is plotted against the total amount of PAH in the vessel, in logarithmic scales. The original values of the spots in the figure are given in Table 3.

III. Effects of Some of Members in Citric Acid Cycle and Related Substances

1) Acetate: It is well known that there is an intimate relation between pyruvate metabolism and acetate.

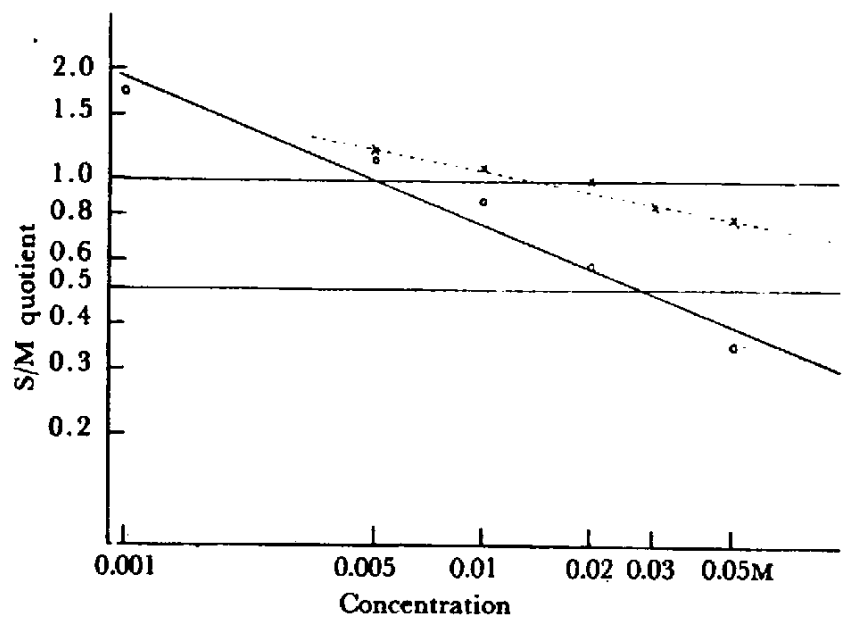

FiG. 6. Effect of concentration of acetate and glucose on the $S / M$ quotient. (0.2 $\mu \mathrm{M}$ of total PAH)

$-\longrightarrow$ Acetate : $\log y=-0.920-0.399 \log x$

$\times \ldots \times$ Glucose : $\log y=-0.349-0.188 \log x$

The result with acetate is given in Fig. 6. It stimulates the accumulation of PAH at low concentrations and the $\mathbf{S} / \mathbf{M}$ quotient becomes proportionally to a power of its concentration smaller than unity, similarly to the case of pyruvate.

The extent of stimulation is somewhat lower than obtained by the previous workers at $25^{\circ} \mathrm{C}$ and 100 cycles/minute. The discrepancy between these results obtained at different temperatures is thought to be apparent, if the degree of stimulation becomes lower at higher temperatures as was seen with pyruvate.

2) Glucose: Glucose is one of the precursors of pyruvate in the energy source of citric acid cycle. The result is given in Fig. 6, and is in accordance with the data obtained by Gross $e t$ al. (5), in spite of the difference between the temperatures of observation. The $S / M$ quotient is almost equal to unity over the range of observed concentrations and the inhibitory effect at high concentrations was not remarkable. 
If glucose would affect on the PAH accumulation due to its aftinity to components of the slices, the effect should be temperature-sensitive as was seen with pyruvate. 'The comparison between this experiment and that of Cross $t$ al. shows no evidence for the sensitivity. Therefore. the obligatory biochemical reactions in utilization of glucose seem to be almost independent on the PAH accumulation.

3) Succinate: The competitive inhibition from accumulation of PAH might be thought to relate intimately with citric acid cycle rather than with the energy source. Succinate is an interesting reagent since it is a member of a metabolic intermediates in citric acid cycle and has been observed mainly its inhibitory effect.

The observation was conducted at lower concentrations, as the inhibition is so intense, that under the concentrations taken for the above mentioned reagents, the accumulation is almost entirely suppressed. The result is given in Fig. 7, which is similar to the data on pyruvate, except that under a given SM quotient, the concentration of succinate is about a tenth of that of pyruvate, that is, the inhibitory action of succinate is ten times

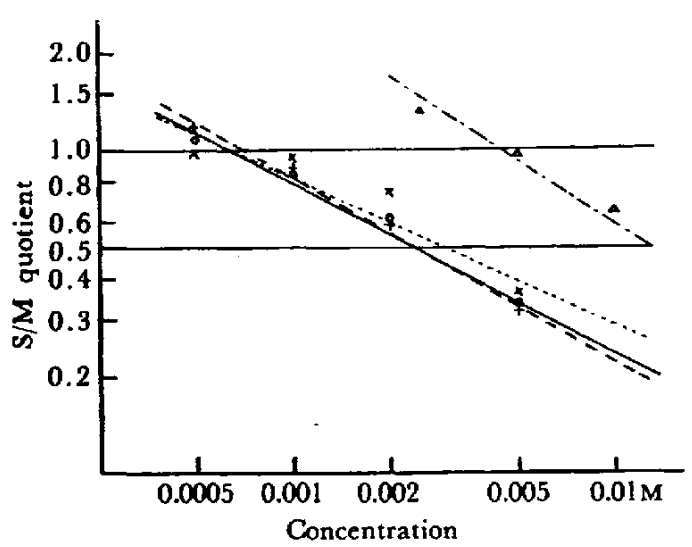

FiG. 7. Effect of concentration of succinate, a-ketoglutarate, and malonate on the $\mathrm{S} / \mathrm{M}$ quotient. (0.2 $\mu \mathrm{M}$ of total PAH)

$\mathrm{O}-$ Succinate : $\log \mathrm{y}=-1.658-0.520 \log \mathrm{x}$

$+--\alpha$ K-etoglutarate : $\log y=-1.754-0.554$ $\log x$

$\times \quad$ Malonate : $\log y=-1.452-0.457 \log x$ A-.. Pyruvate : as large as that of pyruvate. It might be emphasized that in spite of its marked inbibitory effect at low concentrations it shows also stimulation and $S / M$ quotient greater than unity.

4) $\alpha$-Ketoglutarate: This reagent was used under the similar considerations to those described about succinate, and gave a similar result to that of succinate, as shown in Fig. 7. The linhibitory effect was ten times of pyruvate and at low concentrations the stimulation was also observed.

5) Malonate: Malonate has a similar structure of molecule to that of succinate and competes with suc-

TABLE 4. Comparison of malonate with succinate.

\begin{tabular}{lcccc}
\hline \multirow{2}{*}{$\begin{array}{c}\text { Concentration } \\
\text { (mole } L \text { ) }\end{array}$} & \multicolumn{2}{c}{ Malonate } & \multicolumn{2}{c}{ Succinate } \\
S/M ratio* & $\mathrm{O}_{2}$-upt.* & S/M ratio* & O.-upt.* $^{*}$ \\
\hline 0.0005 & 0.98 & 1.00 & 1.07 & 1.09 \\
0.001 & 0.94 & 0.99 & 0.84 & 1.14 \\
0.002 & 0.72 & 0.97 & 0.60 & 1.25 \\
0.005 & 0.36 & 0.83 & 0.33 & 1.40 \\
\hline
\end{tabular}

* Relative value on the basis of the control. 
cinate for succinic dehydrogenase. It is interesting to observe the effect of malonate and to examine whether it shows the stimulatory effect similar to that of succinate.

The result obtained is shown in Table 4 and Fig. 7. At higher concentrations the extent of the inhibition from PAH accumulation did not differ markedly from that of succinate. On the contrary, at lower concentrations, where malonate gives little effect on oxygen uptake, it revealed none of stimulatory action which gives higher values of $\mathrm{S} / \mathrm{M}$ quoticnt than unity.

The plot of S/M quotient against malonate concentration seems to give somewhat curved line. However, the deviation from the best straight line was not significant at $5 \%$ level, on account of the greater value of the variance of $S, M$ quotient at the higher concentrations.

\section{ENZYMOLOGICAL INTERPRETATION}

In the preceding chapter, the data have been represented in Freundlich equation, following Cross and Taggart (5). Generally speaking, enzymological data often fit the equation, and the data of this paper also fit the equation satisfactorily, and, furthermore, the curve of the $50 \%$ inhibition concentration obeyed also the equation, giving a conventional representation of a competitive feature of the inhibitory effect of pyruvate with PAH. However, the Freundlich equation for absorption isotherm is of empirical origin and its theoretical interpretation is rather complicated. Accordingly, with the equation, it is not easy to analyze the results concerning pyruvate and related substances which have been suggested to show the similar competition. The interpretation below refers to another approach based on kinetic treatment of enzymatic reaction.

\section{Relation Between PAH Concentrations in Sliccs and in Medium}

A consideration for the enzymological nature of the results might be approved by application of Michaelis-Menten equation (15) to them. However, their equation deals with velocity of reaction and the results to be analyzed concern with the concentration of PAH, i.e. equilibrium states between PAH and the enzyme. It is, therefore, necessary to modify the derivation, while the main hypotheses of theirs are maintained. Let $\mathrm{E}, \mathbf{M}$, $\mathrm{S}$, and ES represent the free enzyme, free PAH, accumulated PAH, and enzyme-PAH complex, respectively, and let $k_{1}, k_{2}$ express the velocity constants for the corresponding steps given below:

$$
E+M \underset{k_{2}}{\stackrel{k_{1}}{\rightleftarrows}} E S
$$

By enzymatic reactions, it is usual that the enzyme-substrate complex, ES, decomposes to the product, $P$, and free enzyme, $E$, and the overall reaction is written:

$$
E+M \rightleftharpoons E S \rightleftharpoons P+E
$$

In this experiment, equation $(l)$ might be enough to deal with the accumulation and excretion of PAH, since the decomposition recovers free PAH, that is, $\mathrm{P}$ in equation (2) is 
supposed to be identical with $\mathbf{M}$.

Let :

$[\mathrm{E}]_{1}=$ total concentration of the enzyme.

[ES = concentration of the enzyme-PAH complex,

$[E]=$ concentration of the free enzyme,

$(M)=$ concentration of the free PAH.

In the equilibrium state of reaction $(I)$,

$$
k_{1}(E)(M)=k_{2}(E S),
$$

where, [ES) is determined experimentally as concentration of the accumulated PAH in slices, [S], while $k_{q} / k_{1}$ is equal to $K$, the dissociation constant of ES. Then:

$$
(E)=K \frac{1}{(M)}
$$

$[E]_{4}$ is determined as concentration of the accumulated PAH at the saturation, $\left[S_{\infty}\right]$, and is equal to the sum of $[\mathrm{ES}$ : and $[\mathrm{E}\}$, that is :

$$
(E)=[E)_{1}-[E S)=\left(S_{\infty}\right)-(S)
$$

Solving (3) and (4) for (S):

$$
[S]=\frac{\left\{S_{\infty}\right]}{1+\{K /[M]\}}
$$

The well known Michaelis-Menten equation of enzymatic reaction velocity is :

$$
v=\frac{V}{1+\left\{K_{m} /[M]\right\}}
$$

following the notation in this paper. The similarity between these two equations is obvious and the assumptions, which have been taken up to here, are common to the both.

The substitutions of $v, V$, and $K_{m}$ in their equation for $\{S\rangle,\left\langle S_{\infty}\right\rangle$, and $K$ in equation (5), respectively, are not the matter of notation, but show the difference in the subjects between the equations mentioned above. In their equation, $v$ and $V$ represent the overall velocity of reaction (2) from left to right and the maximum velocity which is seen at the saturation, respectively, and $\mathrm{K}_{\mathrm{m}}$ represents Michaelis constant, while $\mathrm{K}$ in equation (5), the true dissociation constant of ES to $\mathrm{E}$ and $\mathrm{M}$. And, furthermore, $\mathrm{K}_{\mathrm{m}}$ is derived approximately neglecting the velocity constant of the inverse reaction from the product to enzyme-substrate complex. This approximation was conducted by them at the beginning of the derivation, and by Briggs \& Haldene (16) in mid course of their modified derivation of the equation. On the contrary, the derivation of equation (5) requires none of these subsidiary assumptions.

Lineweaver \& Burk (17) have pointed out that Michaelis-Menten equation may be recast into a linear equation of $1 v$ and $1 . M$; which is cited as equation (f) in Case I in their paper. The application of the similar transformation to (5) gives:

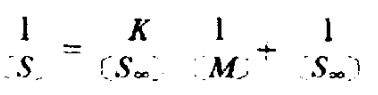

Equation (6) tells us that a plot of $1: \mathrm{S}$ against $1 / \mathrm{M}$ gives a straight line with the intercept on the $1 / S$ axis, $1 S_{x}$, and that on the $1, M$ axis, $1 / K$. 


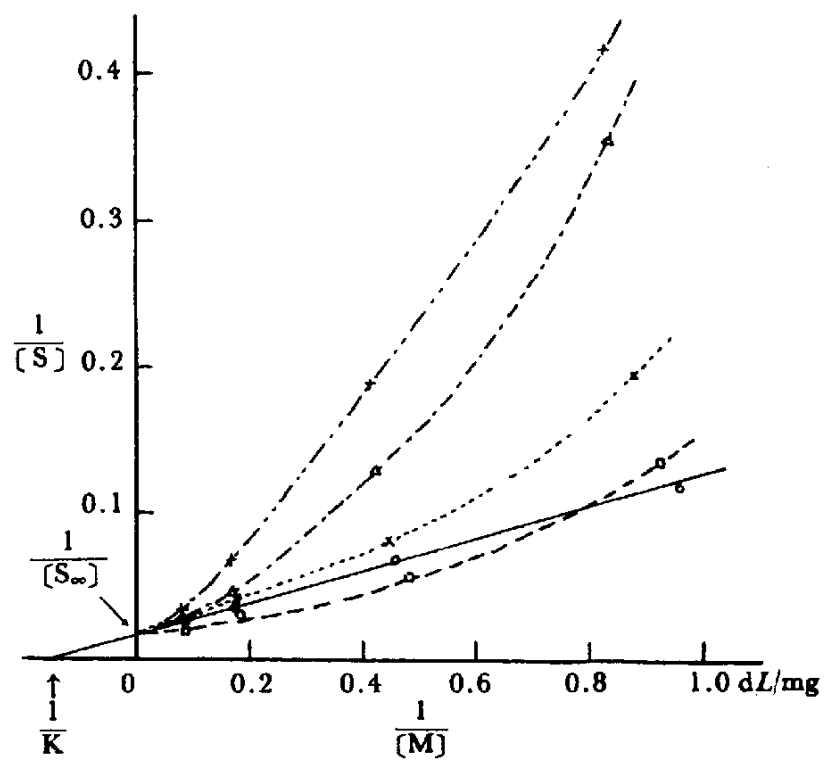

FIG. 8. Relation between PAH content in the slices and in the medium (A).

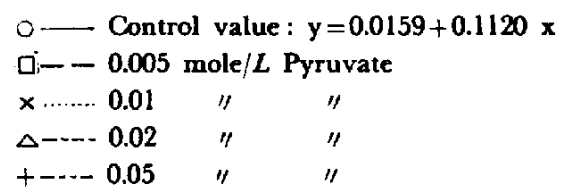

According to equation ( 6 ), the control values in this experiment have been plotted in Fig. 8 and indicated by a full line. The points, the weight of each of which has been estimated on the basis of the variance of 8 examples respectively, are fitted to the line satisfactorily. And the above mentioned model seems to be enough to interpret the accumulation of PAH for the present.

The experimental parameters determined above gave values of $K$ and $\left[S_{*}\right]$, that is : $K=3.63 \times 10^{-4}$ mole $/ L=7.0 \mathrm{mg} / \mathrm{dl}$,
(Variation coefficient : $16.2 \%$ ),
$\left[S_{\infty}\right]=3.24 \times 10^{-8}$ mole $/ L=62.8 \mathrm{mg} / \mathrm{dl}$,
(Variation coefficient : $7.3 \%$ ).

\section{Effect of a Number of Members of Citric Acid Cycle}

A. Effect on the concentration exponent of PAH

The data on the accumulation of PAH in the presence of pyruvate of given concentrations (See Result II) have been replotted in the same way as shown in the previous section and give the dotted lines in Fig. 8. At lower concentrations of pyruvate and higher concentrations of PAH, the deviation from the equation $(6)$ is greater, and it be comes smaller at higher concentrations of the former and lower concentrations of the latter. The stimulatory effect prevails where the deviation is greater, while the inhibitory 
effect is seen where it is smaller. It is well known that competitive inhibition increases the slope of Lineweaver-Burk plot without any concomitant change in its intercept on the vertical axis, while incompetitive inhibition increases both the slope and the intercept. Then, the corresponding changes should be seen in the plot of $1 /(\mathrm{S})$ against $1 /(\mathrm{M})$, according to the substitutions of equation (5) for Michaelis-Menten equation. The plots in Fig. 8, therefore, have a feature of competitive inhibition in spite of their curvature, as has been already pointed out in regard to the 50 ? inhibition plot (See RESULT II, 4).

To interpret their curvature, it must be necessary to generalize the mechanism of competitive inhibition such as presented in a work of Neilands \& Stumpf (18). Following the formulation given before, let $I$ and $M$ represent the substrate, such as pyruvate, and PAH respectively. And the overall reactions may be written with the dissociation constants entered in each reactions :

$$
\begin{aligned}
& E+n M \stackrel{K_{0}^{*}}{\rightleftarrows} E S \\
& E+p I \stackrel{K_{i}^{p}}{\rightleftarrows} E I
\end{aligned}
$$

where, $n$ and $p$ are apparent numbers of molecules of $M$ and $I$ respectively, either of which combines with one active site on the enzyme to form the enzyme-PAH or enzymeinhibitor complex. With other symbols as before, in the equilibrium state of both of the reactions $(7)$ and $\left(7^{\prime}\right)$ :

$$
K_{0}{ }^{n}=\frac{[E]\left[M_{i}\right]^{n}}{[E S]}, \quad K_{i}^{p}=\frac{(E)[E]^{n}}{[E I]}
$$

And the concentration of the free enzyme, $[\mathbf{E}]$, is given :

$$
(E]=\frac{\left[S_{\infty}\right]-[E S]-[E I]}{n}
$$

Solving these equations for $1 /[\mathrm{S}]$ :

$$
\frac{1}{[S)^{-}}=\frac{n K^{\prime}}{\left[S_{\infty}\right]} \stackrel{1}{(M)}+\frac{1}{\left[S_{\infty}\right]}
$$

where,

$$
K^{\prime}=K_{0}^{n}\left\{1+\frac{1}{p}\left(\begin{array}{c}
{[I]} \\
K_{i}
\end{array}\right)^{p}\right\}
$$

$\mathrm{K}^{\prime}$ may be called apparent dissociation constant of ES complex in the presence of $I$, and increases with $p$-th power of $[I]$.

If $n=p=1$, equation $(8)$ reduces to the most simple type of competitive inhibition, corresponding to velocity equation (11) in case IV of Lineweaver \& Burk (17).

To estimate $\mathrm{n}$, concentration exponent of $\mathrm{PAH}$ in equation (8), graphically, putting $n=2$ and $n=3 / 2$, the data have been replotted in Fig. $9(n=2)$ and in Fig. $10(n=3 / 2)$. The latter gives a straight line, and it seems as if three molecules of PAH combine with two active sites on the enzyme (The plot of this type corresponds to case II of Lineweaver \& Burk (17)].

The experimental formulae obtained show that pyruvate alters the concentration exponent of $\mathrm{PAH}, \mathrm{n}$, as stated above, and decreases $\left[\mathrm{S}_{-}\right]$. Since, at low concentrations of pyruvate, the decrease in $\left[S_{\infty}\right]$ is negligible, it might not be taken as the characteristic 


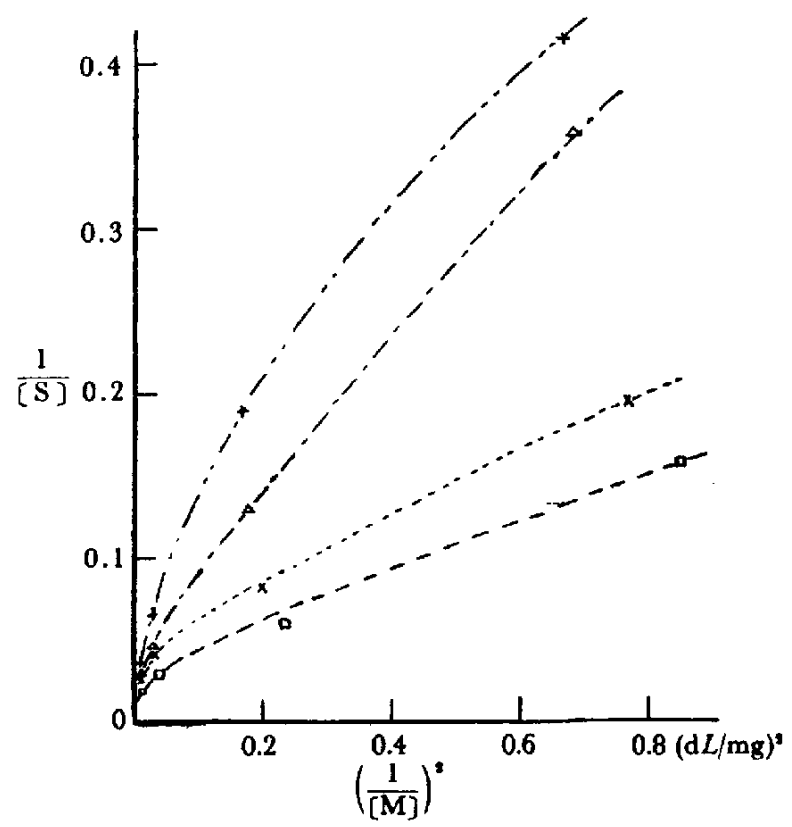

FIG. 9. Relation between PAH content in the slices and in the medium (B).

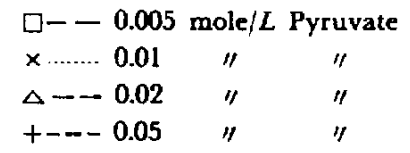

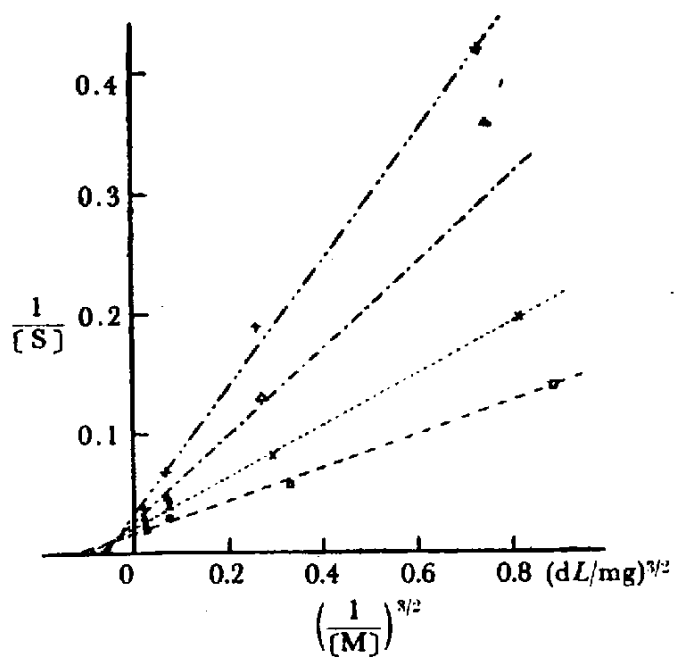

Fig. 10. Relation between PAH content in the slices and in the medium (C).

$\square-0.005$ mole/L Pyruvate: $y=0.0161+0.1378 x$ 0.01 mole/L Pyruvate : $y=0.0209+0.2145 x$ 0.02 mole/ $L$ Pyruvate $y=0.0257+0.3635 x$ 0.05 mole/L Pyruvate : $y=0.0329+0.5306 x$

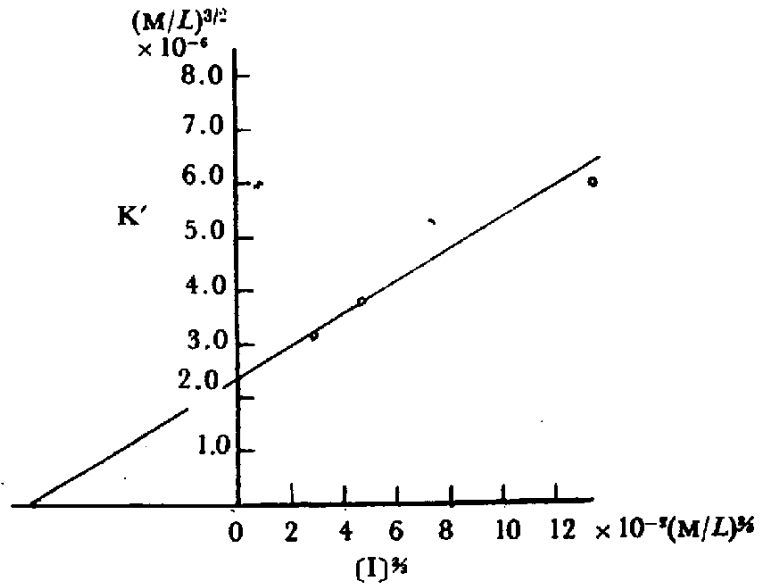

FIG. 11. Relation between the apparent dissociation constant $K^{\prime}$ and the concentration of pyruvate. 
of the stimulatory effect of pyruvate. Except these two, the feature of inhibitory effect of pyruvate is thought to be the same with that of ordinary competitive inhibitors.

In addition, the intercept on the $1 / \mathrm{M}$ ? axis is equal to $-1 / \mathrm{nK}^{\prime}$ and a plot of $\mathrm{K}^{\prime}$ thus obtained against $I$ has been shown in Fig. 11, where $p$ in equation $(9)$ is equal to $2 / 3$, and the intercept gives $\mathrm{K}_{0}{ }^{n}$ and the slope gives $\mathrm{K}_{0}{ }^{n} / \mathbf{p} \mathrm{K}_{i}{ }^{p}$. The apparent dissociation constant of enzyme-PAH complex extraporated to the absence of pyruvate, $\mathrm{K}_{0}{ }^{n}$, and the dissociation constant of enzyme-pyruvate complex, $\mathrm{K}_{i}$, were estimated graphically to be :

$$
\begin{aligned}
& K_{o}^{n}=2.40 \times 10^{-n}(\operatorname{mole} / L)^{s, 2} \\
& K_{i}{ }^{p}=1.17 \times 10^{-1}(\mathrm{~mole} / L)^{3 / 3}
\end{aligned}
$$

On the basis of these examination, it may be concluded that the characteristics of the stimulatory effect of pyruvate are the alterations of the concentration exponent of PAH in the dissociation constant of enzyme-PAH complex and the dissociation constant itself.

\section{B. The concentration exponent's of the members}

In the preceding section, it has been concluded that the member in citric acid cycle, such as pyruvate, affects the PAH accumulation by altering the concentration exponent of PAH in the dissociation constant of the enzyme-PAH complex. In this section, the effect of concentrations of such members on the PAH accumulation under a given concentration of PAH will be treated.

Since the experimental data on the relation between S/M quotient of PAH and concentration of the member added fit Freundlich equation as demonstrated in Resurts IIII, it would not seem unfruitful to compare these data with theoretical relations derived from equation $(8)$ and $(9)$, examining validity of these equations.

Let $S_{o} / M_{o}$ represent the $S_{i} M$ ratio in the absence of pyruvate, and from equation (5),

$$
\frac{\left[S_{0}\right]}{\left[M_{0}\right]}=\frac{\left[S_{\infty}\right]}{K+\left[M_{0}\right]}
$$

$\mathrm{S} / \mathrm{M}$ ratio in the presence of pyruvate is given in the following equation by recasting equation (8),

$$
\frac{S}{M}=\frac{\left(M^{n-1}\left(S_{\infty}\right)\right.}{n K^{\prime}+\left(M^{n}\right.}
$$

here, $\left[S_{\infty}\right]$ is assumed to be independent on $[I]$. Let $R$ represent $S / M$ quotient, then :

$$
\frac{l}{R}=\frac{\left.\left(S_{0}\right) / K M_{0}\right)}{\left.(S) / K M_{i}\right)}=\frac{n K^{\prime}+\left\langle M^{n}\right.}{(M)^{n-1}\left\{\left(M_{0}\right)+K\right\}}
$$

Substituting equation (9):

$$
=\frac{\left.n K_{o}{ }^{n}+[M)^{n}+\left(n K_{0}^{n}\right] p K_{i}^{p}\right)\left\{L^{p}\right.}{\left[M_{j}^{n-1}\left\{K+\left[M_{0}\right]\right\}\right.}
$$

Introducing $\mathrm{a}$ and $\mathrm{b}$ to equation (II), as constants given below,

where,

$$
\frac{I}{R}=a+\frac{C T^{p}}{b}
$$

$$
a=\frac{n K_{0}^{n}+M^{n}}{M^{n-1}\left\{K+M_{0}\right\}}
$$




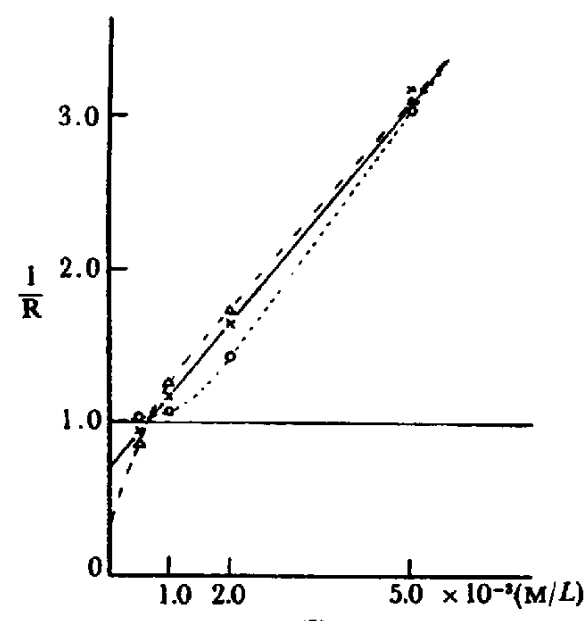

[I]

FIG. 12. Relation between $S / M$ quotient and concentration of the inhibitors (A).

$$
\begin{aligned}
& \mathbf{x}-\text { Succinate } \\
& \Delta--\alpha \text {-Ketoglutarate } \\
& 0 \text {....... Malonate }
\end{aligned}
$$

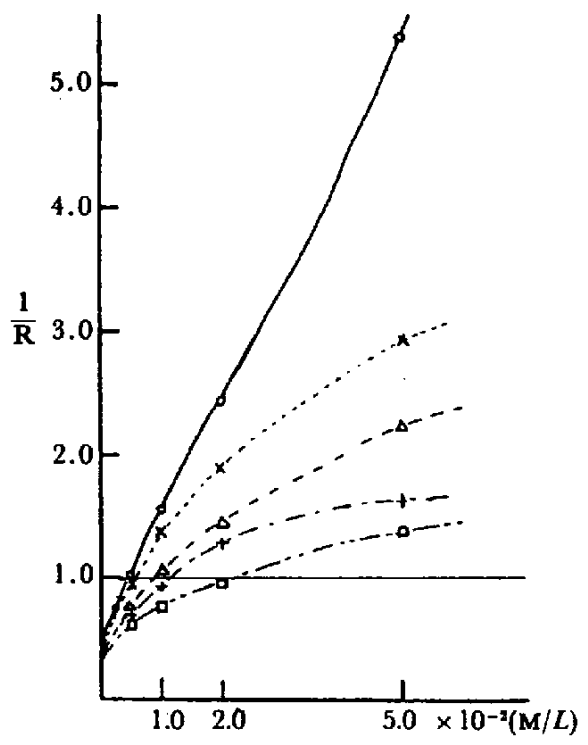

[I]

FIG. 13. Relation between $S / M$ quotient and concentration of the inhibitors (B).

$$
\begin{aligned}
& \mathbf{R}=\mathbf{S} / \mathbf{M} \text { quotient } \\
& \mathrm{I}=\text { Concentration of pyruvate } \\
& 0-0.2 \mu \mathrm{M} \text { of total PAH }\left(37.5^{\circ} \mathrm{C}\right) \\
& \times \ldots . .0 .4 \text { " " " " }(") \\
& \Delta-\cdots-1.0 " \text { " " " }(") \\
& +--2.0 \text { " " " " (") } \\
& \text { 口-- } 0.2 \text { " " " }(25.0 \mathrm{C})
\end{aligned}
$$

$$
b=\frac{\left[M_{i}^{n-1}\left\{K+\left(M_{0}\right]\right\} p K_{i}^{p}\right.}{n K_{*}^{*}}
$$

According to equation (12), $1 / R$ has been plotted against $I$, in Fig. 12 (succinate, $\alpha$-ketoglutarate and malonate), Fig. 13 (pyruvate), and Fig. 14 (acetate and glucose). The plot for succinate gives a straight line, and the exponent $p$ in equation (12) may be unity. This value of $p$ might show that one molecule of succinate combines with one active site on the enzyme in the presence of PAH. And the intercept on the $1 / R$ axis, which is less than unity, shows the stimulatory effect of succinate.

For the members which gave curved line in the above mentioned figures, the concentration exponent $p$ have been graphically determined, that is, $p=2$ for malonate (Fig. 15), $p=2 / 3$ for pyruvate (Fig. 16) and $\alpha$-ketoglutarate (Fig. 17), and $p=1 / 2$ for acetate (Fig. 18). The obtained value of $p$ for pyruvate here is in accord with the value obtained before based on Fig. 11. The experimental formulae of the lines are given in Table 5.

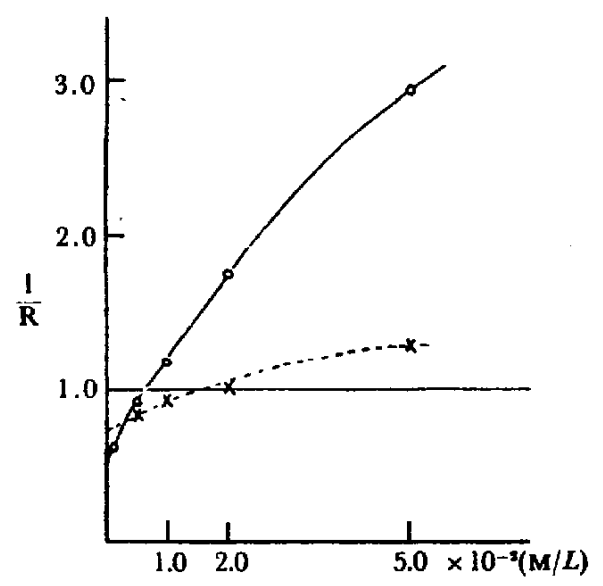

[I]

FG1. 14. Relation between S/M quotient and concentration of the inhibitors (C).

$$
\begin{aligned}
& \mathbf{R}=\mathbf{S} / \mathbf{M} \text { quotient } \\
& I= \text { Concentration of inbibitor } \\
& \mathrm{O}- \text { Acetate } \\
& \times \quad \text { Glucose }
\end{aligned}
$$




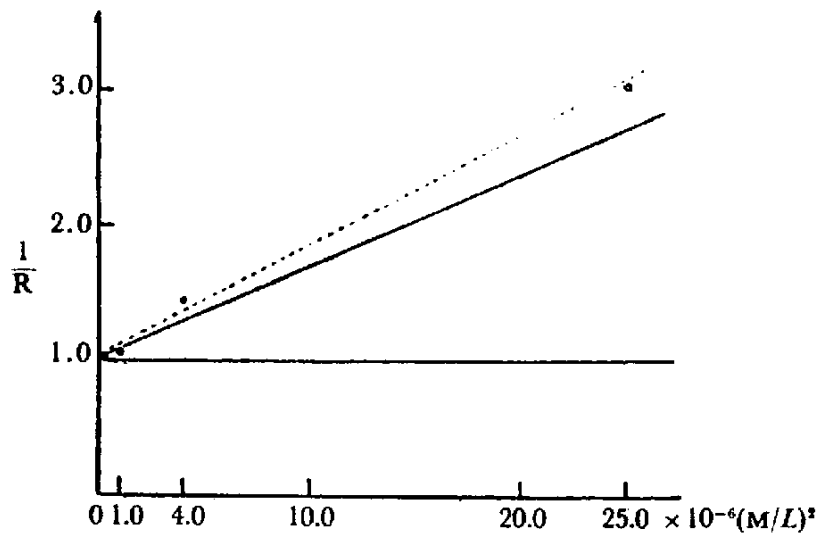

[I]"

FIG. 15. Linear plot of $S / M$ quotient versus concentration of the inhibitors. (A. Malonate)

- obtained by weighting each of the mean values of $1 / R$ obtained without weighting

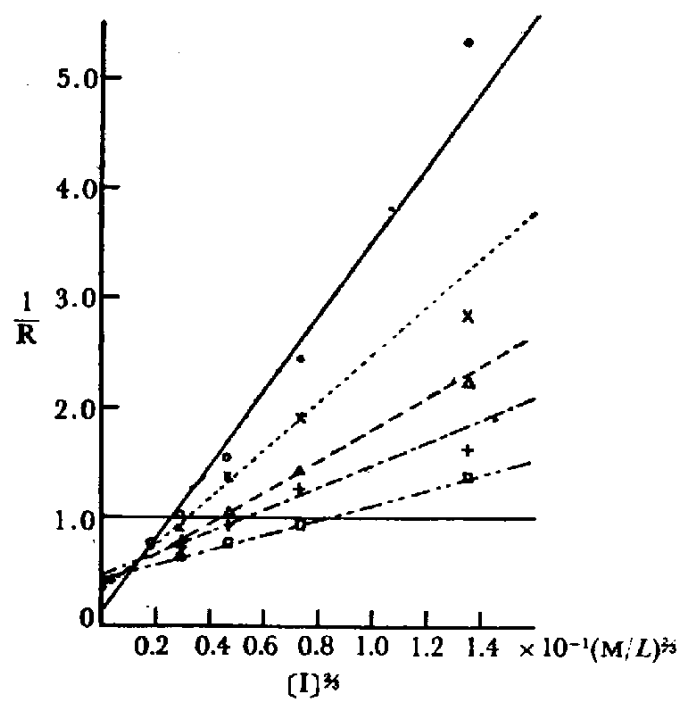

Fig. 16. Linear plot of $S / M$ quotient versus concentration of the inhibitors. (B. Pyruvate) $0-0.2 \mu \mathrm{M}$ of total PAH $\left(37.5^{\circ} \mathrm{C}\right)$

$\times \ldots . .0 .4 "$ " " $"$ "

$\triangle-1.0 "$ " 1 " " " (")

+--- 2.0 " " " " (")

口-- 0.2 " " " " $\left(25.0^{\circ} \mathrm{C}\right)$

Note: The lines were obtained by weighting each of the mean values of $1 / R$.

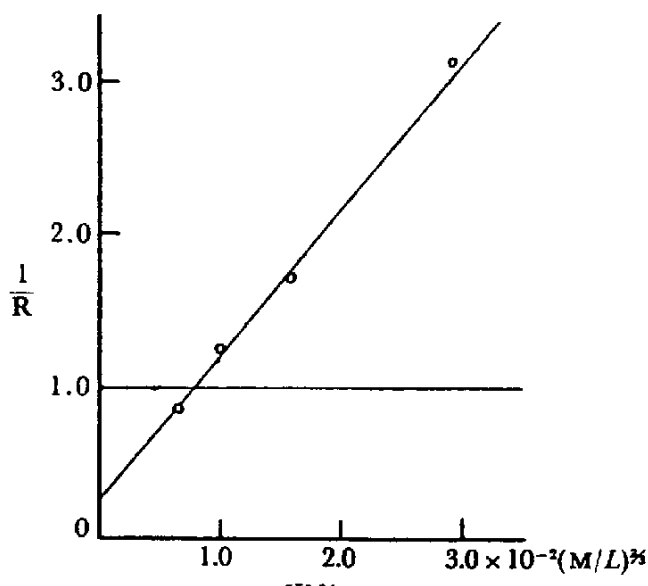

[I] $\%$

FIG. 17. Linear plot of $\mathrm{S} / \mathrm{M}$ quotient versus concentration of the inhibitors. (C. $\alpha$ Ketoglutarate) 
The value of the constant $a$ in equation (12) which happens to be less than unity indicates the stimulatory action of the member. Since $(M)$ and $\left(\mathrm{M}_{\mathbf{a}}\right]$ in equation $(13)$ are nearly equal with each other, as shown in Fig. 8, over the concentration range tested, the constant $\mathbf{a}$ is equal to unity, when $\mathrm{n}=$ 1 and $\mathrm{K}=\mathrm{K}_{0}$, as in a usual case of simple competitive inhibition. And when $\mathrm{nK}_{e}{ }^{n}$ is less than $\mathrm{K}\left(\mathbf{M}_{\mathbf{0}}\right)^{n^{-1}}$, a is less than unity, indicating stimulatory effect. It is meaningless to compare $\mathbf{K}_{\mathbf{0}}{ }^{n}$, apparent dissociation constant of

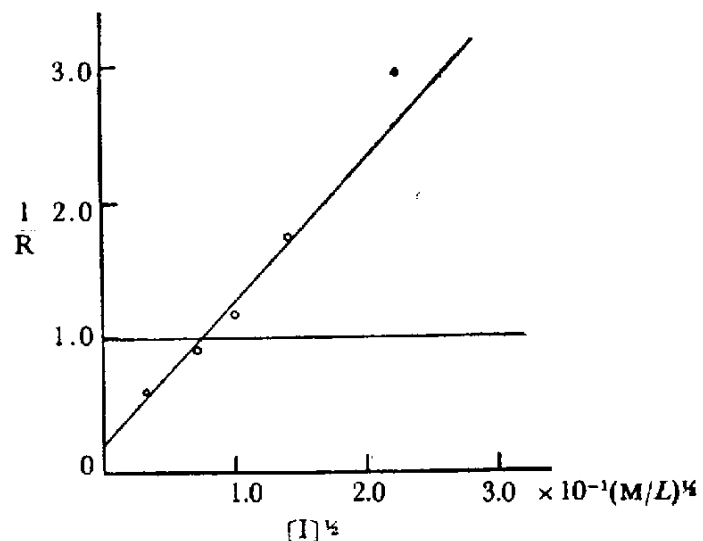

FiG. 18. Linear plot of $S / M$ quotient yersus concentration of the inhibitors. (D. Acetate) the enzyme-PAH complex extraporated

TABLE 5. Effect of concentration of inhibitors on $\mathbf{S} / \mathbf{M}$ quotient.

\begin{tabular}{|c|c|c|c|}
\hline Inhibitor & $\begin{array}{c}\text { Total PAH } \\
(\mu \mathrm{M})\end{array}$ & Temperature & $\begin{array}{l}\text { Experimental } \\
\text { formula* }\end{array}$ \\
\hline Pyruvate & 0.2 & $37.5^{\circ} \mathrm{C}$ & $y=0.133+33.08 x^{3 / 3}$ \\
\hline " & 0.4 & $"$ & $y=0.302+21.55 x^{3 / 3}$ \\
\hline$"$ & 1.0 & $y$ & $y=0.362+14.06 x^{3 / 3}$ \\
\hline$"$ & 2.0 & " & $y=0.484+9.80 x^{3}$ \\
\hline$"$ & 0.2 & $25.0^{\circ} \mathrm{C}$ & $y=0.444+6.89 x^{2} i^{3}$ \\
\hline Acetate & 0.2 & $37.5^{\circ} \mathrm{C}$ & $y=0.205+10.56 \times$ \\
\hline Succinate & $"$ & " & $y=0.696 \div 481.8 x$ \\
\hline$\alpha$-Ketoglutarate & $"$ & " & $y=0.246-97.81 x^{23}$ \\
\hline Malonate & " & $y$ & $y=1.002 \div 68.314 x^{2}$ \\
\hline
\end{tabular}

* $y$ : the inverse of $S / M$ quotient

$x$ : concentration of inhibitor in mole $L$

to $I=0$, directly with $K$, dissociation constant of it in the absence of $I$, as the former is usually different in its dimension from the latter. And after taking $n K_{o}{ }^{n}$ and $K \backslash M_{o} j^{n-1}$, the comparison would get its meaning, if the above mentioned conditions of approximation were filled.

\section{Derivation of Freundlich equation}

As has been mentioned in the previous section, the data fit equation (13), and a in the equation is unity when malonate competes with PAH, and less than unity with the other members.

Recasting equation (13) into a logarithmic form,

$$
\log \{(1 / R)-a\}=p \log (I,-\log b
$$


A plot of $\{(/ / R)-a\}$ against $I$ in logarithmic scale gives a straight line, as shown in Fig. 19. The slope gives $p$, that is, $p=1$ for succinate, $p=2.10 \fallingdotseq 2$ for malonate, $p=0.68$ $\fallingdotseq 2 / 3$ for pyruvate, $p=0.70 \fallingdotseq 2 / 3$ for $\alpha$-ketoglutarate, $p=0.49 \fallingdotseq 1 / 2$ for acetate. These values are in accord with those obtained in the previous section.

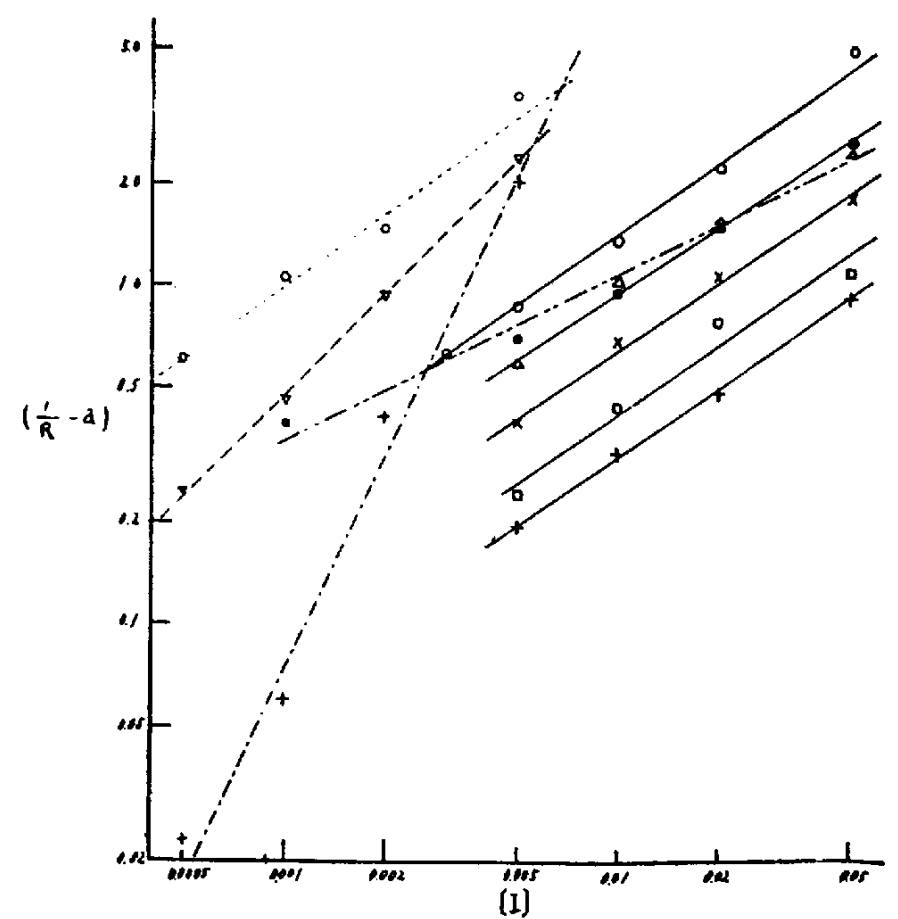

FIG.. 19. Relation between $(1 / \mathrm{R})-\mathrm{a}$ and the concentration of inhibitors.

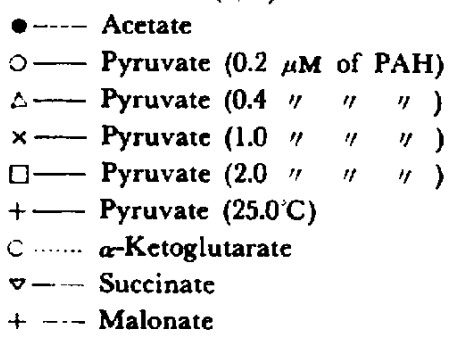

When $1 / \mathrm{R}$ is greater than a, equation (15) gives:

$$
\log R=\log b-p \log (I)
$$

This formula is identical with experimental formulae of Freundlich type, obtained in Result I-III.

\section{DISCUSSION}

The results obtained in this experiment showed that a group of members in citric acid cycle have the stimulatory as well as the inhibitory effects on the PAH accumulation by kidney slices. The discussion below refers to some aspects on the mechanism of these effects. 


\section{1) The stimulatory effect}

According to the mathematical formulation undertaken in the previous chapter, the stimulatory effect has been represented as the alterations of some of parameters in the equation of the competitive inhibition. And the effects of the tested members fit this formulation without exception. Even acetate, on which Cross at al. have noticed only the stimulatory effect at $25^{\circ} \mathrm{C}$ and within a concentration range up to $0.01 \mathrm{~mole} / L$, showed at $37.5^{\circ} \mathrm{C}$ the evident inhibitory effect at the higher concentrations, while the stimulatory effect was somewhat slighter within the same concentration range which was taken by them. These results might indicate that the stimulatory effect was essentially accompanied with the inhibitory effect. Of course, the inhibitory effect of acetate might be expected to become so feeble at $25^{\circ} \mathrm{C}$, in consideration of the data on pyruvate, that it would become comparable with experimental error and it would seem to be lost apparently.

As the stimulatory effect of acetate is especially marked at $25^{\circ} \mathrm{C}$, Taggart of al. (5-12) have suggested that acetate was "a rate-limiting cellular component of the PAH transport mechanism." But the mechanism itself has not been established, since it has not been succeeded to confirm that the stimulatory effect should be due to $p$-aminoacetylation. In conclusion, they have supposed that acetate, or its counterparts in other transport systems, shared in the activation process through interaction with energy-rich phosphates and the energy available for such purpose constitutes another limiting factor in transport (5). The effect of acetate has been also detected in vivo (6) and its protective effects against various inhibitors have been found in viue and in vitro $(8,11,19)$.

Now, in this experiment it was confirmed that besides acetate, such members of citric acid cycle as succinate and $\alpha$-ketoglutarate also had stimulatory action, which had not yet been known. And these action were represented in a common formulation. Therefore, the emphasis which they have placed on acetate does not seem to be necessary. The citric acid cycle is an important energy-yielding mechanism, and the alteration of $\mathbf{K}$ and $\mathbf{n}$ in equation (9) suggests that the stimulation is due to increase in efficiency of enzyme or activation of latent enzyme by promotion of the cycle.

Shideman et al. (19) have stated that the effect of acetate did not cause by promotion of oxidative metabolism, on the basis of the data on oxygen uptake. In this experiment also the increase in oxygen uptake did not correlate with the amount of accumulated PAH. This is possible, because the amount of the accumulated PAH does not depend on the velocity of the accumulation. That is, as mentioned in ENzYMOL. INTERPRET. I, if the overall reaction is written as :

$$
E+M \rightleftarrows E S \rightleftarrows P+E
$$

where, $P$ and $M$ represent the same substance, $P A H$, and the amount of the accumulated PAH corresponds to the amount of its complex with enzyme and, probably, the amount staying in renal tubulus, then these amounts themselves are expected to be constant under various magnitudes of velocity of the transport corresponding to the extent of energy supply. The oxygen uptake, therefore, is not required to increase with the amount of 
accumulated PAH, and the data of the non-parallelism between these two factors should not be taken to indicate the absence of the correlation between oxygen uptake and PAH transport mechanism.

The alteration of activity of the enzyme which accumulates PAH on itself, might be thought to cause the stimulatory effect at low concentrations of pyruvate and the like members, on the basis of these speculations.

\section{2) The inhibitory effect}

There have been few works on the inhibitory effect of such members of citric acid cycle as pyruvate and acetate, though the effect has been well known with succinate and $\alpha$-ketoglutarate. Besides them, the effect of malate and fumarate has been known, and it has been confirmed in viro together with the stimulatory effect of acetate, but the inhibitory effect itself has discussed by few workers. Shideman and Rene (20) have been discussed the relation between the inhibition of succinic oxidase system and the inhibitory effect of malonate, dehydroacetate, cincophen, and chlorguanide from PAH accumulation, and stated that the energy necessary to the accumulation was supplied through oxidation of succinic acid.

Succinate and $\alpha$-ketoglutarate have been known as the most powerful inhibitors among the above cited members. Both of these two substrates and malonate have shown the extent of inhibition ten times as large as that of pyruvate, in this experiment. Since the effect of pyruvate was competitive as has been concluded in RESULT II and ENzYMOL. INTERPRET. II, the inhibitory action of succinate and the like members, which have similar structure to that of pyruvate in regard to being carboxylic acids, is thought to be competitive with PAH for enzyme.

The inhibitory concentration exponents, 'p's given in equation (12)], of pyruvate, acetate, and $\alpha$-ketoglutarate. which were not equal to unity, may indicate that the effects of these members reveal themselves having been modified by any subsidiary enzyme or participation in another concurrent metabolic cycle. Then, the apparent number of molecules of the inhibitor which combine with one active site on the principal enzyme may differ from unity. On the contrary, the exponent of succinate is equal to unity, and this substrate seems to combine directly with the principal enzyme.

It is suggested, therefore, that the principal enzyme has a specific relation to succinate and bears indirect relation to pyruvate, acetate, and $\alpha$-ketoglutarate. Furthermore, these relations, direct and indirect, may show that there is an intimate relation between citric acid cycle and the PAH accumulation.

After these interpretation, it seems natural to discuss about the enzymes which have affinity with succinate, especially succinic dehydrogenase participating in citric acid cycle.

It is well known that malonate competes with succinate for succinic dehydrogenase, and this competition is appropriate to the explanation of the data. Succinic dehydrogenase has been detected its abundant presence in renal tubular cell except descending legs of Henle's loop $(21,22)$. The enzyme has been also known to be inhibited by such substances 
as $\mathrm{Cu}$ ion and iodoacetate which are inhibitors of SH radical, and by malonate and fumarate also (23). S/M ratio decreases in the presence of iodoacetate and fluoroacetate, as well as the above mentioned substances. As stated previously, Shideman \& Rene (20) have been indicated that succinic oxidase system affects the accumulation of PAH in kidney slice, and Beyer et al. (4) suggested that succinic dehydrogenase has an intimate relation to excretion of phenol red based on the confirmation of the inhibitory actions of $\mathbf{H g}$ ion, quinone, and phenylhydrazine, etc.

The participation of succinic dehydrogenase should be concluded carefully, because it is possible that malonate inhibits another enzyme. For example, according to Massey (24), fumarase is also inhibited by malonate and succinate to the same extent. The enzyme, however, is not affected by acetate and iodoactate, both of which inhibit succinic dehydrogenase. These inaccessibility shows that the enzyme does not enter in the accumulation of PAH.

While it might be necessary to include into consideration other metabolic cycles in which succinate enters, the decisive confirmation of the enzyme and the cycle, which accumulate PAH, is thought to be difficult. However, it is sufficiently probable that certain enzyme, participating in citric acid cycle, perhaps succinic dehydrogenase, directly affects the accumulation, as a group of members in that cycle has been confirmed to compete with PAH.

If these considerations are valid, the excretion of $\mathrm{PAH}$ is thought to be conducted by dehydrogenation followed by exchange of anions. And this exchange is expected to be conducted in a similar way with the exchange of cations, such as seen by the excetion of $\mathrm{K}^{+}$, $\mathrm{Na}^{+}$, and $\mathrm{H}^{+}$. Because the PAH accumulation is known to be inhibited by substituting various anions for $\mathrm{Cl}$ ion (9), and PAH shows osmotic diuresis which is effected by other anion species (25). And the exchange of $\mathrm{PAH}$ and $\mathrm{Cl}$ ion is supported also by the observation of the effect of mercurial diuretics on the accumulation $(5,27)$. These diuretics inhibit succinic dehydrogenase and reabsorption of $\mathrm{Cl}$ ion $(25,26,28)$.

Another standpoint of interpretation might be given, when the inhibition is supposed to be apparent one. For example, it might be caused by energy balance. The substrate might be subjected to a change to synthetize various products. These concurrent syntheses interfere with each other as a given amount of energy divided among them. Though the syntheses have been detected in kidney $(29,30)$, it is not probable that the inhibition is due to these interference, since acetate which is the most reactive among the substrates rather stimulates than inhibits the accumulation.

Besides this type of inhibition, it might be supposed that the inhibition is depend on phosphorylation, such as the inhibition by phloridzin (5). Then, the substrate, utilization of which requires phosphorylation inevitably, such as glucose, should be expected to affect the accumulation evidently, but the data with glucose does not seem to be consistent with this idea. 


\section{SUMMARY}

The effects of a number of members in citric acid cycle and related substances on the accumulation of PAH by rat kidney slices are observed. Pyruvate, acetate, succinate, and $\alpha$-ketoglutarate exhibit stimulation as well as inhibition. The results have been enzymologically interpreted and the velocity equations of Lineweaver-Burk have been modified to be applied to the equilibrium of enzyme-PAH complex formation. The stimulation is represented as the alteration of parameters in the equation of competitive inhibition.

It is supposed that the stimulatory effect is due to increase in efficiency of enzyme and activation of latent enzymes and the inhibitory effect is due to the competition between these members and PAH for the enzyme, which participates in the PAH transport mechanism. The enzyme is thought to act in citric acid cycle and suggested to be succinic dehydrogenase.

Acknowledgement. In conclusion the author expresses his gratitude to Prof. Dr. S. Kozawa for his helpful suggestions and criticisms on this work.

\section{REFERENCES}

1) SMith, H.W., Goldring, W. ANd Chasis, H.: J. clin. Inver. 17, 263 (1938)

2) FoRSTER, R.P.: Srience 109, 65 (1948)

9) TAggART, J.V. AND FORSTER, R.P.: Am. J. Physio!. 161, 167 (1950)

4) BeYer, K.H., Pa:NTer, R.M. ANd Wiebelhau \&, V.D.: Ibid. 161, 259 (1950)

5) CROSS, R.J. AND TAggarT, J.V.: Mid. 161, 181 (1950)

6) MUdGe, G.H. AND Taggarr, J.V.: Ibid. 161, 191 (1950)

7) Mudge, G.H. AND TaggarT, J.V.: Ibid. 161, 173 (1950)

8) Farah, A., Graham, G. ANd Koda, F.: J. Pharmacol. 108, 410 (1953)

9) Taggart, J.V., Sillerman, J. And Trayner, E.M.: Amer. J. Physid. 167, 531 (1951)

10) Carhart, E., Koda, F. ANd Farah, A.: Proc. Sor. Expor. Biol. \& Mod. 85, 248 (1954)

11) Farah, A., KodA, F. AND Frazer, M.: J. Pharmard. 113, 169 (1954)

12) SChachter, D. AND Freinkel, N.: Amet. J. Physid. 167, 531 (1951)

13) Bratton, A.C. AND Marshall, E.K.: J. bid. Chem. 123, 537 (1939)

14) DEMiNG, W.E. : Statistical .Adjurtment of Data Ed. 1, Iwanami-shoten (1950) (transtated in Japanase)

15) M:Chaelis, L. AND MENTEN, M.L. : Biochem. Z. 29, 333 (1913)

16) BRiGg:, E. AND HALDEN, J.B.S.: Biochom. $J$. 19, 338 (1925). Cited from (18)

17) LiNewraver, H. AND BURK, D.: J. Am. Chem. Soc. 56, 658 (1934)

18) Neilands, J.B., STUmPF, P.K. AND STANier, R.Y.: Outlines of Enzyme Chemistry, Ed. 1, 97, Asakura shoten (1956) (trandated in Japanese)

19) Sh.deman, F.E., Rathbun, R.C. And Stoneman, F.: Amer. J. Physiol. 170, 31 (1952)

20) SHIDEMAN, F.E. AND RENE, R.M. : Ibid. 166, 104 (1951)

21) WACHSTE'N : Gited from SAKAI, F.: Shizen, 11, No. 4, 59 (1956)

22) Sel.gman, A.M. AND RUTenburg, A.M.: Science 113, 317 (1951)

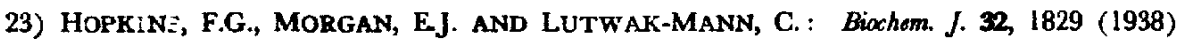


24) Massey, I.: Ibid. 55, 172 (1953)

25) Pitts, R.F. AND Sartorius, O.W. Pharmard. Rei. 2, 161 (1950)

26) Yoshitoshi, Y.: Nippon-rinshô 14, 1769 (1956)

27) Koishi, $T$.: In press

28) HaNdley, C.A. AND LAviK, P.S.: $J$. Pharmaco!. 100,115 (1950)

29) WeIL-MAHLERBE, H. : Biochem. J. 2,2276 (1938)

30) TENG, C.T.: .Arch. Biachcm. \& Biophysics 48, 409 (1954) 